\title{
On the Separability of GDoF Region for Parallel Gaussian TIN Optimal Interference Networks
}

\author{
Hua Sun and Syed A. Jafar \\ Center for Pervasive Communications and Computing (CPCC) \\ University of California Irvine, Irvine, CA 92697
}

\begin{abstract}
It has been shown recently by Sun et al. that in a $K$ user parallel Gaussian interference network, if over each sub-channel, for each user the desired signal strength is no less than the sum of the strengths of the strongest interference from this user and the strongest interference to this user (all signal strengths measured in $\mathrm{dB}$ scale), then separate coding over each sub-channel and treating interference as noise (TIN) is sufficient to achieve the sum generalized degrees of freedom (GDoF), subject to a mild invertibility condition [1]. In this work, we show that the weighted sum GDoF is similarly separable, i.e., separate coding and TIN is sufficient to achieve the weighted sum GDoF, subject to a similar mild invertibility condition. This is proved by translating the weighted GDoF optimization problem to the sum GDoF problem of a class of compound parallel Gaussian interference networks, giving rise to new weighted GDoF outer bounds that are strictly stronger than what is implied by the sum GDoF bounds obtained previously.
\end{abstract}

\section{INTRODUCTION}

There is much recent interest in the capacity of Gaussian interference networks in the regime where the simplest scheme, where each transmitter uses point to point Gaussian codebook with power control and each receiver "treats interference as noise" (TIN), is information theoretically optimal. The information theoretic optimality of TIN is considered from the exact capacity perspective in [2], [3], [4], [5] where such a TIN optimal regime is identified. However, either due to the difficulty of characterizing the exact capacity or because exact optimality of TIN is indeed rare, the regime that is identified so far is very small, limiting its significance in practice. On the other hand, one would expect a much larger regime where TIN is approximately optimal. Such a regime may be useful for broader insights into the optimality of TIN and may be also more tractable. Based on this intuition, recent work has explored the optimality of TIN by relaxing the metric from exact capacity to approximate capacity, specifically in terms of the generalized degrees of freedom (GDoF).

The starting point of this work is the result by Geng et al. in [6], where an elegant TIN optimality condition is identified under which TIN is sufficient to achieve the whole GDoF region (also the capacity region to a constant gap). The TIN optimality condition is that in a $K$ user Gaussian interference network, for each user the desired signal strength is no less than the sum of the strengths of the strongest interference from this user and the strongest interference to this user

This work is supported in part by funding from ONR and by NSF grants CCF-1317351 and CCF-1319104. (all signal strengths measured in $\mathrm{dB}$ scale). More recently, [1] considers the $K$ user parallel Gaussian TIN optimal interference network, whereby each sub-channel satisfies the TIN optimality condition. It is shown that the sum GDoF of the parallel network can be achieved by separate coding and TIN over each sub-channel, subject to a mild invertibility condition. The main motivation of this work is to go beyond the sum GDoF to the GDoF region, and in particular to answer the following question: Is separate TIN over each sub-channel still optimal for the GDoF region of the $K$ user parallel network collectively, given that each sub-channel individually is TIN optimal?

As the GDoF region can be described by the weighted sum GDoF for all choices of weights, we focus on the weighted sum GDoF problem. We show that any weighted GDoF optimization (that is, any supporting hyperplane of the GDoF region) of the parallel network can, without loss of optimality, be solved separately over each sub-channel and the optimal value for each sub-channel can be achieved by TIN, subject again to a mild invertibility condition similar to the one identified in [1]. The main difficulty which limited our previous work to sum GDoF is that, unlike the case without parallel channels studied by Geng et al. where it is shown that sum-GDoF bounds are sufficient to characterize the entire GDoF region, with parallel channels it turns out that sum GDoF bounds alone are not sufficient to describe the region achieved by separate TIN (an example is given in [1]). This is essentially because the GDoF region is not polymatroidal. The new insight that allows us to overcome this difficulty in this work is that the weighted GDoF optimization problem can be translated into the sum GDoF problem of a class of compound parallel Gaussian interference networks. For any given vector of weights, we are able to define a corresponding compound parallel Gaussian interference network such that the weightedsum GDoF of the original parallel network is equal to the sum GDoF of this compound parallel network. This allows us to use the insights from [1] to find the sum GDoF value and show that it is achieved by separate TIN on each subchannel.

\section{System Model, Definitions, AND PRELiminaries}

\section{A. Gaussian Interference Network and GDoF Framework}

We retain the channel model of [1], represented as follows. Consider the $K$ user real Gaussian interference network, with 
$M$ parallel sub-channels, described as

$$
\begin{array}{r}
Y_{k}^{[m]}(t)=\sum_{i=1}^{K} \operatorname{sign}\left(\tilde{h}_{k i}^{[m]}\right) \sqrt{P^{\alpha_{k i}^{[m]}}} X_{i}^{[m]}(t)+Z_{k}^{[m]}(t) \\
\forall k \in[K] \triangleq\{1,2, \ldots, K\}, m \in[M] \triangleq\{1,2, \ldots, M\}
\end{array}
$$

where at each time index $t$, over the $m$-th sub-channel, $X_{i}^{[m]}(t)$ is the transmitted symbol from Transmitter $i, Y_{k}^{[m]}(t)$ is the received signal observed at Receiver $k, \operatorname{sign}\left(\tilde{h}_{k i}^{[m]}\right) \in\{-1,1\}$ indicates the real channel from Transmitter $i$ to Receiver $k$ is negative or positive, $\sqrt{P^{\alpha_{k i}^{[m]}}}$ is its channel magnitude, and $Z_{k}^{[m]}(t) \sim \mathcal{N}(0,1)$ is the additive white Gaussian noise (AWGN) at Receiver $k$. Noise processes are i.i.d over time and sub-channels. All symbols are real. We call the exponent $\alpha_{k i}^{[m]}$ the channel strength level and as done in [6], [1], we assume $\alpha_{k i}^{[m]}$ values are non-negative (the negative values are mapped to zero). All channel coefficients are fixed over time. Perfect channel knowledge is available everywhere.

At Transmitter $i$, an independent message $W_{i}$ uniformly distributed over the index set $\left\{1,2, \ldots, 2^{n R_{i}}\right\}$ is mapped to the codeword $\left[X_{i}^{[1]}(1), \ldots, X_{i}^{[M]}(1), X_{i}^{[1]}(2), \ldots, X_{i}^{[M]}(n)\right]$ over $n$ time slots, and is subject to the average power constraint, $\frac{1}{n} \sum_{t=1}^{n} \sum_{m=1}^{M}\left|X_{i}^{[m]}(t)\right|^{2} \leq 1$. At Receiver $k$, the received signal $\left[Y_{k}^{[1]}(1), \ldots, Y_{k}^{[M]}(1), Y_{k}^{[1]}(2), \ldots, Y_{k}^{[M]}(n)\right]$ is used to produce the estimate $\hat{W}_{k}$ of the message $W_{k}$. The probability of error for Receiver $k$ is given by the probability that $\hat{W}_{k}$ is not equal to $W_{k}$. A rate tuple $\mathbf{R}(P)=$ $\left(R_{1}(P), R_{2}(P), \ldots, R_{K}(P)\right)$ is said to be achievable if we have an encoding and decoding mapping such that the probability of error for each receiver approaches zero as $n$ approaches infinity. The capacity region $\mathcal{C}(P)$ is the closure of the set of all achievable rate tuples.

We define the (convex) GDoF region as the collection of weighted sum GDoF problems in (2), shown at the top of the next page, and the sum GDoF value as $\mathcal{D}_{\Sigma}=\max _{\mathcal{D}} \sum_{k=1}^{K} d_{k}$.

If the following TIN optimality condition is satisfied

$\alpha_{i i}^{[m]} \geq \max _{j: j \neq i}\left\{\alpha_{j i}^{[m]}\right\}+\max _{k: k \neq i}\left\{\alpha_{i k}^{[m]}\right\}, \quad \forall i, j, k \in[K], m \in[M]$

the network (sub-channel) is referred to as a TIN optimal network (sub-channel) and in this work, we focus exclusively on TIN optimal networks, i.e., the condition holds throughout.

\section{B. Weighted Directed Graph Representation}

A directed graph is defined for each sub-channel of the parallel interference network. As all definitions and notations to be introduced are associated with one specific sub-channel, the sub-channel index superscript is omitted in this subsection for compactness. The directed graph representation of one subchannel of the parallel interference network consists of $K$ vertices, $V_{1}, V_{2}, \cdots, V_{K}$, one for each user. Since the vertices correspond directly to users, we will also refer to them as users. For all $(i, j) \in[K] \times[K]$, there is a directed edge $e_{i j}$ from user $j$ to user $i$, with weight $w\left(e_{i j}\right)$ defined as $w\left(e_{i j}\right)=\alpha_{i j}$ if $i \neq j$ and $w\left(e_{i j}\right)=0$ if $i=j$.
We are particularly interested in the notion of cycles on this directed graph. We define a cycle, $\pi$, as a cyclically ordered subset of users, without repetitions. The set of all cycles is denoted as $[\Pi]$. The cardinality of a cycle, denoted as $|\pi|$ is the number of users that it involves. $|\pi|=\sum_{V_{k} \in \pi} 1, \forall \pi \in[\Pi]$. A cycle with only one user is a trivial cycle. Two cycles $\pi_{p}, \pi_{q}$, are said to be disjoint if they contain no common user, denoted as $\pi_{p} \cap \pi_{q}=\phi$.

Introducing a slight abuse of notation in the interest of conciseness, the same cycle, $\pi$, can also be equivalently represented as a set of edges representing a closed path where no user is visited more than once. The weight of a cycle, denoted as $w(\pi)$, is the sum of the weights of all the edges traversed in completing the cycle, i.e., $w(\pi)=\sum_{e_{i j} \in \pi} w\left(e_{i j}\right), \forall \pi \in[\Pi]$. Note that the weight of a trivial cycle is zero. Intuitively, the weight of a cycle is the accumulation of the strengths of interference terms encountered in the cycle.

Cyclic Partition: A subset of the set of all cycles, $\Pi \subset[\Pi]$, is said to be a cyclic partition if $\pi_{p} \cap \pi_{q}=\phi, \forall \pi_{p}, \pi_{q} \in \Pi$ and $\sum_{\pi \in \Pi}|\pi|=K$. In other words, a cyclic partition is a disjoint cyclic cover of the $K$ users.

Cyclic Partition Bound: For any cyclic partition $\Pi$, define the corresponding cyclic partition bound, $\mathcal{D}_{\Sigma}^{\Pi}$, as $\sum_{k=1}^{K} d_{k} \leq$ $\sum_{k=1}^{K} \alpha_{k k}-w(\Pi)$, where $w(\Pi)=\sum_{\pi \in \Pi} w(\pi)$ is the net weight of the cyclic partition, representing the total interference encountered in this partition. We also call $w(\pi)$ cycle bound, then cyclic partition bound is merely a collection of disjoint cycle bounds. Since there are many cyclic partitions, each of which gives rise to a cyclic partition bound, let us denote the tightest of these bounds as the best cyclic partition bound, $\mathcal{D}_{\Sigma}^{\Pi *}$. A cyclic partition that produces the best cyclic partition bound is labeled an optimal cyclic partition, and denoted by $\Pi^{*}$. For example, when $K=6$, one possible cyclic partition is $\Pi=\{\{1,3,5\},\{4,2\},\{6\}\}$ which decomposes the users into three cycles, such that each user is represented in exactly one cycle. The corresponding cyclic partition bound is $\sum_{k=1}^{6} d_{k} \leq \sum_{k=1}^{6} \alpha_{k k}-\left(\alpha_{13}+\alpha_{35}+\alpha_{51}\right)-\left(\alpha_{42}+\alpha_{24}\right)-(0)$.

Participating Edge: Edge $e_{i j}$ is a participating edge for the cyclic partition $\Pi$ if $i \neq j$ and $e_{i j} \in \pi$ for some $\pi \in \Pi$.

Cyclic Predecessor: Under cyclic partition $\Pi$, the cyclic predecessor for user $k$ is user $\Pi(k)$, if $e_{\Pi(k) k}$ is a participating edge for $\Pi$. Note that if user $k$ belongs to a trivial cycle in $\Pi$ then $\Pi(k)=\phi$.

Participating Input and Output Levels $\left(X_{i, u}, Y_{k, u}\right)$ : For any sub-channel, we define participating input levels $X_{i, u} \triangleq$ $0 . X_{i,(1)}, \ldots, X_{i,\left(n_{\Pi(i) i}\right)}, n_{\Pi(i) i}=\left\lfloor\frac{1}{2} \alpha_{\Pi(i) i} \log _{2} P\right\rfloor$ to be the bits levels that are below the decimal point, sent from Transmitter $i$ and observed at its predecessor Receiver $\Pi(i)$. The received signal levels resulting from all interfering $X_{i, u}$ plus additive Gaussian noise are defined as the participating output levels $Y_{k, u} \triangleq \sum_{i=1, i \neq k}^{K} \operatorname{sign}\left(\tilde{h}_{k i}\right) \sqrt{P^{\alpha_{k i}}} X_{i, u}+Z_{k}$.

Invertibility: One sub-channel is said to be invertible within bounded noise distortion if the mapping from $\mathbf{X}_{u} \triangleq$ $\left(X_{1, u}, \ldots, X_{K, u}\right)$ to $\mathbf{Y}_{u} \triangleq\left(Y_{1, u}, \ldots, Y_{K, u}\right)$ is invertible for an optimal cyclic partition $\Pi^{*}$. Mathematically, we require $H\left(\mathbf{X}_{u} \mid \mathbf{Y}_{u}\right)=o(\log (P))$. 


$$
\mathcal{D}=\left\{\left(d_{1}, \cdots, d_{K}\right) \in \mathbb{R}_{+}^{K}: \forall\left(\mu_{1}, \cdots, \mu_{K}\right) \in \mathbb{R}_{+}^{K}, \quad \mu_{1} d_{1}+\cdots+\mu_{K} d_{K} \leq \limsup _{P \rightarrow \infty}\left[\sup _{\mathbf{R}(P) \in \mathcal{C}(P)}\left[\mu_{1} R_{1}(P)+\cdots+\mu_{K} R_{K}(P)\right] \frac{1}{\log P}\right]\right\}
$$

$$
\begin{aligned}
& \bar{Y}_{i ; p l l}^{[m]}=\operatorname{sign}\left(\tilde{h}_{i i}^{[m]}\right) \sqrt{P^{\alpha_{i i}^{[m]}}} \bar{X}_{i ; p}^{[m]}+\sum_{j=1, j \neq i}^{K} \operatorname{sign}\left(\tilde{h}_{i j}^{[m]}\right) \sqrt{P^{\alpha_{i j}^{[m]}}} \bar{X}_{j ; a_{j}}^{[m]}+\bar{Z}_{i ; p: l}^{[m]}, \forall i \in[K], m \in[M], \quad a_{i+1}=\left\lceil\frac{l}{\mu_{i+2} \mu_{i+3} \cdots \mu_{i-1}}\right\rceil, \\
& a_{j}=\left\lceil\frac{l-\sum_{q=i+1}^{j-1}\left(a_{q}-1\right) \mu_{q+1} \mu_{q+2} \cdots \mu_{i-1}}{\mu_{j+1} \mu_{j+2} \cdots \mu_{i-1}}\right\rceil, j=\{i+2, i+3, \cdots, i-2\}, \quad a_{i-1}=l-\sum_{q=i+1}^{i+K-2}\left(a_{q}-1\right) \mu_{q+1} \mu_{q+2} \cdots \mu_{i-1}
\end{aligned}
$$

Remark: Participating inputs and output levels $\left(X_{i, u}, Y_{k, u}\right)$ and associated invertibility properties $\left(H\left(\mathbf{X}_{u} \mid \mathbf{Y}_{u}\right)\right)$ are defined respective to some cyclic partition, though this dependency is not explicitly shown in the notation. The referred cyclic partition is understood from the context when these notations appear. We mention that the invertibility property is mild. For a detailed discussion, we refer to Section 5.3.2 in [1].

To consider the weighted sum GDoF problem, we translate it to the sum GDoF problem of another network, defined next. Suppose we are considering maximizing $\mu_{1} d_{1}+\cdots+\mu_{K} d_{K}$ for positive integer weights $\mu_{i} \in \mathbb{Z}_{+}, i \in[K]$.

\section{Replicated Compound Network}

In the replicated compound network, Transmitter $i$ is replicated $\mu_{i}, i \in[K]$ times. Each replicated transmitter $k \in[K]$ has $\prod_{i=1, i \neq k}^{K} \mu_{i}$ compound receivers. An example with $K=$ $3,\left(\mu_{1}, \mu_{2}, \mu_{3}\right)=(3,2,1)$ is shown in Figure 1.

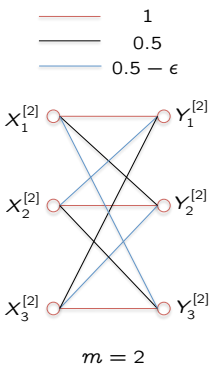

(a)

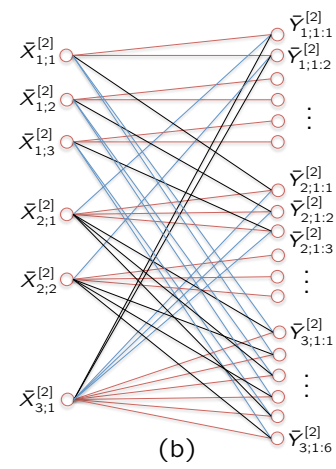

(b)

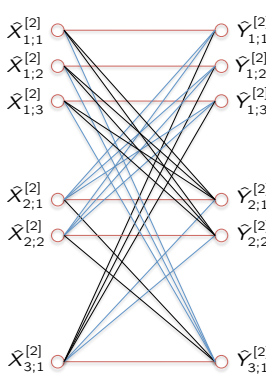

(c)
Fig. 1. (a) The second sub-channel of an network, (b) the corresponding replicated compound sub-channel and (c) the corresponding auxiliary sub-channel (to be used in later proof). The channel strength level is indicated by the color of each link. To avoid cluttering the figure, in (b), only the channels to the receivers associated with the first replicate of each transmitter are shown.

The input output relationship of the replicated compound network is described as (3), shown at the top of this page, where over the $m$-th sub-channel, the transmitted symbol from the $p$-th replicate of Transmitter $j$ is denoted as $\bar{X}_{j ; p}^{[m]}, \forall p \in$ $\left\{1, \ldots, \mu_{j}\right\}$, the received signal and AWGN observed at the $l$-th compound receiver associated with the $p$-th replicate of Transmitter $i$ are represented by $\bar{Y}_{i ; p: l}^{[m]}$ and $\bar{Z}_{i ; p: l}^{[m]}, \forall p \in$ $\left\{1, \ldots, \mu_{i}\right\}, l \in\left\{1, \ldots, \prod_{j=1, j \neq i}^{K} \mu_{j}\right\}$, respectively. The indices are interpreted modulo $K$. We set $\bar{Z}_{i ; p: l}^{[m]}=Z_{i}^{[m]}$. In words, the network is designed such that each compound receiver sees one and only one interference from each interfering replicated transmitter, without repetition.

For the replicated compound network, each transmitter has an independent message for its compound receivers. Capacity region and associated notions are defined similar to those of the original network. Specifically, we denote the GDoF tuple as $\left(\bar{d}_{1 ; 1}, \ldots, \bar{d}_{1 ; \mu_{1}}, \bar{d}_{2 ; 1}, \ldots, \bar{d}_{K ; \mu_{K}}\right)$ and the sum GDoF value as $\overline{\mathcal{D}}_{\Sigma}=\max _{\overline{\mathcal{D}}}\left(\sum_{k=1}^{K} \sum_{j=1}^{\mu_{k}} \bar{d}_{k ; j}\right)$, where $\overline{\mathcal{D}}$ is the GDoF region of the replicated compound network.

The usage of the replicated compound network lies in the following lemma, which reduces the weighted sum GDoF problem to the sum GDoF problem.

Lemma 1: The weighted GDoF of the original network is equal to the sum GDoF of the replicated compound network, i.e., $\mu_{1} d_{1}+\cdots+\mu_{K} d_{K}=\overline{\mathcal{D}}_{\Sigma}$.

The proof is based on using the coding schemes of the original network in the replicated compound network and vice versa. Details are presented in the extended paper [7].

After this reduction, the problem is still not immediately solved, as the sum GDoF of a parallel TIN optimal compound interference network is generally open. But our constructed replicated compound network contains some symmetry that allows us to solve its sum GDoF value analytically. To find $\overline{\mathcal{D}}_{\Sigma}$, it is helpful to introduce the weighted directed graph representation for the replicated compound network, similar to that of the original network.

We have one directed graph for each compound state of the replicated sub-channel. One compound state is defined by keeping only one compound receiver for each replicated transmitter. Consider one sub-channel and one compound state where the received signals are $\bar{Y}_{1 ; 1: l_{1 ; 1}}, \cdots, \bar{Y}_{1 ; \mu_{1}: l_{1 ; \mu_{1}}}, \bar{Y}_{2 ; 1: l_{2 ; 1}}, \cdots, \bar{Y}_{K ; \mu_{K}: l_{K ; \mu_{K}}}$. Note that the sub-channel index superscript is omitted for brevity. The directed graph consists of $\sum_{k=1}^{K} \mu_{k}$ vertices, $\bar{V}_{1 ; 1}, \cdots, \bar{V}_{1 ; \mu_{1}}, \bar{V}_{2 ; 1}, \cdots, \bar{V}_{K ; \mu_{K}}$, one for each user. For all $i, j \in[K], p_{i} \in\left\{1, \cdots, \mu_{i}\right\}, p_{j} \in\left\{1, \cdots, \mu_{j}\right\}$, there is a directed edge $\bar{e}_{i_{p_{i}} j_{p_{j}}}$ from user $\left(j ; p_{j}\right)$ to user $\left(i ; p_{i}\right)$, with weight $w\left(\bar{e}_{i_{p_{i}} j_{p_{j}}}\right)$ defined as $w\left(\bar{e}_{i_{p_{i}} j_{p_{j}}}\right)=\alpha_{i j}$ if $i \neq j, a_{j}=p_{i}$ and 0 otherwise, where $a_{j}$ is defined according to the index of the compound receiver $l_{i ; p_{i}}$. In words, the weight of an edge 
is equal to the strength of the interfering link.

A cycle, $\bar{\pi}$, is a cyclically ordered subset of users, without repetitions. The set of all cycles is denoted as $[\bar{\Pi}]$. The cardinality of a cycle, denoted as $|\bar{\pi}|$ is the number of users that it involves. $\bar{\pi}$, can also be equivalently represented as a set of edges representing a closed path where no user is visited more than once. The weight of a cycle, denoted as $w(\bar{\pi})$, is the sum of the weights of all the edges traversed in completing the cycle.

Cyclic Partition: A subset of the set of all cycles, $\bar{\Pi} \subset[\bar{\Pi}]$, is said to be a cyclic partition if $\bar{\pi}_{p} \cap \bar{\pi}_{q}=\phi, \quad \forall \bar{\pi}_{p}, \bar{\pi}_{q} \in$ $\bar{\Pi}, \sum_{\bar{\pi} \in \bar{\Pi}}|\bar{\pi}|=\sum_{k=1}^{K} \mu_{k}$.

Cyclic Partition Bound: For any cyclic partition $\bar{\Pi}$, define the corresponding cyclic partition bound, $\overline{\mathcal{D}} \bar{\Sigma}$, as $\sum_{k=1}^{K} \sum_{j=1}^{\mu_{k}} \bar{d}_{k ; j} \leq \sum_{k=1}^{K} \mu_{k} \alpha_{k k}-w(\bar{\Pi})$, where $w(\bar{\Pi})=$ $\sum_{\bar{\pi} \in \bar{\Pi}} w(\bar{\pi})$.

We denote the tightest of cyclic partition bounds for all cyclic partitions as the best cyclic partition bound, $\overline{\mathcal{D}} \bar{\Sigma}{ }_{\Sigma}$. A cyclic partition that produces the best cyclic partition bound is labeled an optimal cyclic partition, and denoted by $\bar{\Pi}^{*}$.

Participating Edge: Edge $\bar{e}_{i_{p_{i}} j_{p_{j}}}$ is a participating edge for the cyclic partition $\bar{\Pi}$ if $i \neq j$ and $\bar{e}_{i_{p_{i}} j_{p_{j}}} \in \pi$ for some $\bar{\pi} \in \bar{\Pi}$.

Cyclic Predecessor: Under cyclic partition $\bar{\Pi}$, the cyclic predecessor for user $\left(k ; p_{k}\right)$ is user $\bar{\Pi}\left(k ; p_{k}\right)$, if $\bar{e}_{\bar{\Pi}\left(k ; p_{k}\right) k_{p_{k}}}$ is a participating edge for $\bar{\Pi}$.

Participating Input and Output Levels $\left(\bar{X}_{i ; \underline{p}_{i}, u}, \bar{Y}_{k ; p_{k}, u}\right)$ : We define the participating input levels as $\bar{X}_{i ; p_{i}, u} \triangleq 0 . \bar{X}_{i ; p_{i},(1)}, \ldots, \bar{X}_{i ; p_{i},\left(\bar{n}_{\bar{\Pi}\left(i ; p_{i}\right) i_{p_{i}}}\right)}, \bar{n}_{\bar{\Pi}\left(i ; p_{i}\right) i_{p_{i}}}=$ $\left\lfloor\frac{1}{2} w\left(\bar{\Pi}\left(i ; p_{i}\right) i_{p_{i}}\right) \log _{2} P\right\rfloor$. We define the participating output levels as the linear combination of the interfering participating input levels plus noise, $\bar{Y}_{k ; p_{k}, u} \triangleq \sum_{i=1, i \neq k}^{K} \operatorname{sign}\left(\tilde{h}_{k i}\right) \sqrt{P^{\alpha_{k i}}} \bar{X}_{i ; a_{i}, u}+Z_{k}$, where $a_{i}$ is defined with the index of the compound receiver $l_{k ; p_{k}}$.

Invertibility: The compound state of one sub-channel is said to be invertible within bounded noise distortion if the mapping from $\overline{\mathbf{X}}_{u} \triangleq\left(\bar{X}_{1 ; 1, u}, \ldots, \bar{X}_{K ; \mu_{K}, u}\right)$ to $\overline{\mathbf{Y}}_{u} \triangleq$ $\left(\bar{Y}_{1 ; 1, u}, \ldots, \bar{Y}_{K ; \mu_{k}, u}\right)$ is invertible for an optimal cyclic partition $\bar{\Pi}^{*}$. Mathematically, we require $H\left(\overline{\mathbf{X}}_{u} \mid \overline{\mathbf{Y}}_{u}\right)=o(\log (P))$. One sub-channel is said to be invertible if the compound state whose best cyclic partition bound is the tightest among all compound states is invertible.

\section{GDOF REGION}

We first review the result in [1] on the sum GDoF of $K$ user parallel Gaussian TIN optimal interference networks.

Theorem 1: (Theorem 5 in [1]) In a $K$ user parallel Gaussian TIN optimal interference network with $M$ sub-channels, if each sub-channel is individually invertible, then the sum GDoF value of the parallel Gaussian interference network is achieved by separate TIN over each sub-channel. Further, the sum GDoF value is given by the sum of best cyclic partition bound over each sub-channel, i.e., $\mathcal{D}_{\Sigma}=\sum_{m=1}^{M} \mathcal{D}_{\Sigma}^{\Pi^{[m] *}}=$ $\sum_{m=1}^{M}\left[\sum_{i=1}^{K} \alpha_{i i}^{[m]}-w\left(\Pi^{[m] *}\right)\right]$.

We now move from sum GDoF to GDoF region. Interestingly, when we only have one sub-channel, the sum GDoF bounds proved in Theorem 1 are sufficient to describe the GDoF region, which can be achieved by TIN. This is proved by Geng et al. in [6]. We restate this result in the following, as it gives us the achievable TIN region for each sub-channel by itself. For a parallel network, the Minkowski sum of the TIN region for each sub-channel is the achievable region by separate TIN.

Theorem 2: (Theorem 1 in [6]) In a $K$ user Gaussian TIN optimal interference network with one sub-channel, power control and treating interference as noise achieve the entire GDoF region. Moreover, the GDoF region is given by all sum GDoF bounds given in Theorem 1 for all subsets of users.

The situation is different as we proceed from single subchannel case to multiple parallel sub-channels. We have the following observation from Theorem 10 in [1], "the region described by all tight sum-GDoF bounds for all subsets of users is in general not the same as the region achievable by separate TIN over each sub-channel." Then it is noted [1] that "either the separate TIN achievable region is not tight or we need more than sum-rate bounds." Here we show that the latter is true, i.e., the sum GDoF bounds do not suffice.

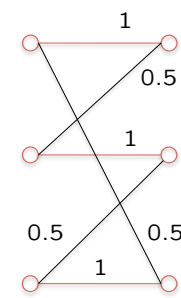

$m=1$

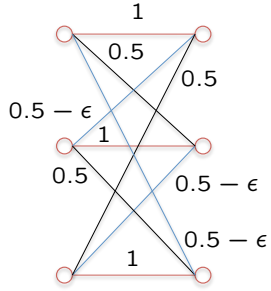

$m=2$
Fig. 2. A $K=3$ user Gaussian interference network with 2 subchannels. The channel strength level is indicated for each link. Each sub-channel is TIN optimal and invertible [1].

To be specific, we consider an example, which was introduced in [1] to show the above notes. Consider a $K=3$ user Gaussian TIN optimal interference network with $M=2$ subchannels, depicted in Figure 2. We set $\epsilon=0.01$. It is shown in [1] that each sub-channel is invertible such that Theorem 1 applies. The sum GDoF bounds proved in Theorem 1 for all subsets of users are the following.

$$
\begin{aligned}
d_{1} & \leq 1+1=2 \\
d_{2} & \leq 1+1=2 \\
d_{3} & \leq 1+1=2 \\
d_{1}+d_{2} & \leq 1.5+(1+\epsilon)=2.5+\epsilon \\
d_{2}+d_{3} & \leq 1.5+(1+\epsilon)=2.5+\epsilon \\
d_{3}+d_{1} & \leq 1.5+(1+\epsilon)=2.5+\epsilon \\
d_{1}+d_{2}+d_{3} & \leq 1.5+1.5=3
\end{aligned}
$$

We now consider the tightest possible bound for $3 d_{1}+2 d_{2}+$ $d_{3}$, from only sum GDoF bounds, (4) - (10). That is

$$
(4)+(7)+(10) \Rightarrow 3 d_{1}+2 d_{2}+d_{3} \leq 7.5+\epsilon
$$


However, it turns out that the tightest bound for $3 d_{1}+2 d_{2}+$ $d_{3}$ is

$$
3 d_{1}+2 d_{2}+d_{3} \leq 7+3 \epsilon
$$

which is tighter than (11). A proof of (12) can be found in the extended paper [7]. Here we show how to get the RHS value of this bound. As we will show that separate TIN is sufficient to achieve any weighted sum GDoF, the tightest bound for $3 d_{1}+2 d_{2}+d_{3}$ could be evaluated separately for each subchannel and then add them up. That is

$3 d_{1}+2 d_{2}+d_{3}=3 d_{1}^{[1]}+2 d_{2}^{[1]}+d_{3}^{[1]}+3 d_{1}^{[2]}+2 d_{2}^{[2]}+d_{3}^{[2]}$

where $\left(d_{1}^{[m]}, d_{2}^{[m]}, d_{3}^{[m]}\right) \in \mathbb{R}_{+}^{3}, m \in\{1,2\}$ denotes the GDoF tuple of the $m$-th sub-channel by itself. They satisfy the following constraints given by Theorem 2 .

$$
\begin{aligned}
d_{1}^{[1]} & \leq 1 \\
d_{2}^{[1]} & \leq 1 \\
d_{3}^{[1]} & \leq 1 \\
d_{1}^{[1]}+d_{2}^{[1]} & \leq 1.5 \\
d_{2}^{[1]}+d_{3}^{[1]} & \leq 1.5 \\
d_{3}^{[1]}+d_{1}^{[1]} & \leq 1.5 \\
d_{1}^{[1]}+d_{2}^{[1]}+d_{3}^{[1]} & \leq 1.5 \\
d_{1}^{[2]} & \leq 1 \\
d_{2}^{[2]} & \leq 1 \\
d_{3}^{[2]} & \leq 1 \\
d_{1}^{[2]}+d_{2}^{[2]} & \leq 1+\epsilon \\
d_{2}^{[2]}+d_{3}^{[2]} & \leq 1+\epsilon \\
d_{3}^{[2]}+d_{1}^{[2]} & \leq 1+\epsilon \\
d_{1}^{[2]}+d_{2}^{[2]}+d_{3}^{[2]} & \leq 1.5
\end{aligned}
$$

Thus the tightest bound on $3 d_{1}+2 d_{2}+d_{3}$ based only on sum GDoF bounds for each sub-channel by itself is obtained by the sum of the following two bounds (referring to (12))

$$
\begin{aligned}
(13)+(16)+(19) & \Rightarrow 3 d_{1}^{[1]}+2 d_{2}^{[1]}+d_{3}^{[1]} \leq 4 \\
2 \times(23)+(25) & \Rightarrow 3 d_{1}^{[2]}+2 d_{2}^{[2]}+d_{3}^{[2]} \leq 3(1+\epsilon)
\end{aligned}
$$

Let us now see why linear combinations of sum GDoF bounds are loose. Note that the RHS of the sum GDoF bounds of the parallel network, (4) - (10) are the sum of the corresponding RHS of the sum GDoF of each sub-channel by itself, (13) - (19) and (20) - (26). But in order to get the tightest bound on $3 d_{1}+2 d_{2}+d_{3}$, as shown in (27) and (28), we need two different linear combinations of the sum GDoF bounds of each sub-channel by itself, which is not available from the sum GDoF bounds of the parallel network. The fundamental reason is that the TIN region for each sub-channel is not polymatroidal such that their Minkowski sum is not described fully by the direct sum of corresponding inequalities for each sub-channel.
With some additional effort, we can show that the bound (12) is achievable as well, by separate TIN. In fact, this example on maximizing weighted GDoF is representative of our general result. We will show that any weighted sum GDoF problem can be solved separately over each sub-channel and the optimal value for each sub-channel is achieved by TIN. We state this result in the following theorem.

Theorem 3: In a $K$ user parallel Gaussian TIN optimal interference network with $M$ sub-channels, if each sub-channel of the replicated compound channel is invertible, then the weighted sum GDoF of the parallel Gaussian interference network is achieved by separate TIN over each sub-channel.

The sketch of the proof is given as follows. Lemma 1 translates the weighted sum GDoF problem of the original network to the sum GDoF problem of the replicated compound network. To find the sum GDoF of the replicated compound network, we resort to an auxiliary non-compound network (see Figure 1(c)), which contains all interfering links in all possible states. Such an auxiliary network has the property that its best cyclic partition bound is the same as the replicated compound network and it is further achievable by separate TIN over each sub-channel. Details are given in [7].

\section{CONCLUSION}

For $K$ user parallel Gaussian TIN optimal interference networks, we show that separate TIN over each sub-channel is optimal under a mild invertibility condition, from the perspective of weighted sum GDoF, extending previous work on sum GDoF. As in the sum GDoF case, we conjecture that the invertibility condition is mild, i.e., it holds in almost all cases. Varying the weights of the weighted sum GDoF problem, we are able to cover all supporting hyperplanes of the compact convex GDoF region. But we do not claim the separability of the whole GDoF region as we are not sure the GDoF region is described by a finite number of weighted sum GDoF inequalities and we need to verify the invertibility for the replicated compound network for every possible weights (possibly infinite).

\section{REFERENCES}

[1] H. Sun and S. A. Jafar, "On the Optimality of Treating Interference as Noise for $K$ user Parallel Gaussian Interference Networks," ArXiv http://arxiv.org/abs/1401.2692, Jan, 2014.

[2] X. Shang, G. Kramer, and B. Chen, "A new outer bound and the noisyinterference sum-rate capacity for gaussian interference channels," IEEE Transactions on Information Theory, vol. 55, no. 2, pp. 689-699, Feb. 2009.

[3] A. Motahari and A. Khandani, "Capacity bounds for the gaussian interference channel," IEEE Transactions on Information Theory, vol. 55, no. 2, pp. 620-643, Feb. 2009.

[4] V. Annapureddy and V. Veeravalli, "Gaussian interference networks: Sum capacity in the low interference regime and new outer bounds on the capacity region," IEEE Trans. on Information Theory, pp. 3032-3050, July 2009.

[5] X. Shang, B. Chen, G. Kramer, and H. V. Poor, "Noisy-Interference SumRate Capacity of Parallel Gaussian Interference Channels," IEEE Trans. on Inf. Theory, vol. 57, no. 1, pp. 210-226, 2011.

[6] C. Geng, N. Naderializadeh, S. Avestimehr, and S. Jafar, "On the Optimality of Treating Interference as Noise," ArXiv:1305.4610.

[7] H. Sun and S. A. Jafar, "On the Separability of GDoF Region for Parallel Gaussian TIN Optimal Interference Networks," Available at https://webfiles.uci.edu/huas2/tin\%20weighted\%20isit\%20proof.pdf. 